\title{
Overview of bariatric surgery for the physician
}

\author{
Keng Ngee Hng and Yeng S Ang
}

\begin{abstract}
The worldwide pandemic of obesity carries alarming health and socioeconomic implications. Bariatric surgery is currently the only effective treatment for severe obesity. It is safe, with mortality comparable to that of cholecystectomy, and effective in producing substantial and sustainable weight loss, along with high rates of resolution of associated comorbidities, including type 2 diabetes. For this reason, indications for bariatric surgery are being widened. In addition to volume restriction and malabsorption, bariatric surgery brings about neurohormonal changes that affect satiety and glucose homeostasis. Increased understanding of these mechanisms will help realise therapeutic benefits by pharmacological means. Bariatric surgery improves long-term mortality but can cause long-term nutritional deficiencies. The safety of pregnancy after bariatric surgery is still being elucidated.
\end{abstract}

KEY WORDS: bariatric surgery, obesity, weight loss, diabetes, hormones

\section{Introduction}

Obesity is a worldwide pandemic, ${ }^{1-4}$ with the number of obese children and adolescents increasing alarmingly. ${ }^{5}$ This has serious health and socioeconomic implications due to the attendant increase in related comorbidities. ${ }^{1,2,4,6}$ Obesity causes type 2 diabetes, hypertension, dyslipidaemia, cardiovascular disease, obstructive sleep apnoea, obesity hypoventilation syndrome, cancer, steatohepatitis, gastro-oesophageal reflux, gallstones, pseudotumour cerebri, osteoarthritis, infertility and urinary incontinence. ${ }^{1-6}$ Severe obesity reduces life expectancy by $5-20$ years. ${ }^{1}$

Diet, exercise and drug treatments for severe obesity have been disappointing. ${ }^{1-3,5-12}$ At the present time, bariatric surgery is the only treatment that reliably produces substantial and sustainable weight loss. ${ }^{1-7,9,13}$ It is indicated in people with BMI $>40 \mathrm{~kg} / \mathrm{m}^{2}$ or with $\mathrm{BMI}>35 \mathrm{~kg} / \mathrm{m}^{2}$ in the presence of significant comorbidity. $3,5,7,14$ Bariatric surgery is cost effective, ${ }^{3,6,15}$ achieving weight loss, as well as improvement or resolution of associated comorbidities. ${ }^{1,2,5,6,9,15,16}$ In the past decade, the development of centres of excellence, ${ }^{5,6}$ laparoscopic techniques, ${ }^{2,5,6}$ improved safety profiles ${ }^{2,6,9}$ and better documentation of clinical

Keng Ngee Hng, ${ }^{1}$ specialty registrar in gastroenterology; Yeng S Ang, ${ }^{2}$ consultant gastroenterologist and honorary lecturer

${ }^{1}$ Salford Royal NHS Foundation Trust; ${ }^{2}$ Faculty of Medicine, University of Manchester, Oxford Road, Manchester effectiveness ${ }^{2,6,15}$ have fuelled an increase in the number of procedures performed.

\section{Types of surgery}

Bariatric surgical procedures are traditionally classified as restrictive, malabsorptive or combined according to their mechanism of action. The procedures most commonly performed are laparoscopic adjustable gastric banding and roux-en-y gastric bypass. ${ }^{3,13}$ Sleeve gastrectomy is increasingly performed. ${ }^{2,6,7}$ Biliopancreatic diversion and biliopancreatic diversion with duodenal switch are much more complex and performed infrequently. ${ }^{2,5,17,22}$ Other historical procedures are no longer in common use.

In addition to restriction and malabsorption, recent evidence suggests that neurohormonal changes are an important effect of bariatric surgery. 2,6,7,17,18 Bariatric surgery is only part of the management of severe obesity. Careful patient selection and preparation are extremely important, as are long-term compliance with diet, nutritional supplementation and follow up. ${ }^{2,5,6,19}$

\section{Laparoscopic adjustable gastric banding ${ }^{2}$}

A purely restrictive procedure, laparoscopic adjustable gastric banding ( $\mathrm{LABG}$ ) is the least invasive procedure, is completely reversible and has the lowest mortality. ${ }^{19}$ A silicone inflatable band is placed around the stomach cardia immediately below the gastrooesophageal junction (Fig 1). This is connected to a subcutaneous port that is used for band adjustment. ${ }^{5,6}$ The band compresses the cardia to generate a sense of satiety and reduced appetite, which is thought to be mediated via vagal afferents. ${ }^{2}$

\section{Roux-en-Y gastric bypass}

Roux-en-Y gastric bypass (RYGB) is a combined procedure that is also performed laparoscopically. A $20-30-\mathrm{ml}$ gastric pouch connected to the jejunum forms the Roux limb (Fig 2). The disconnected duodenal limb is anastomosed 75-150 cm along the Roux limb, forming a Y configuration. The distal stomach, duodenum and part of the proximal jejunum are thus bypassed. ${ }^{5-7,20}$ Despite the traditional classification of the this procedure, malabsorption is not significant with the standard RYGB surgery. ${ }^{7}$ In an extended gastric bypass, the Roux limb is lengthened to increase the malabsorptive component. ${ }^{6,20}$

\section{Sleeve gastrectomy}

In sleeve gastrectomy, $60-80 \%$ of the stomach is removed along the greater curvature to leave a restricting 'sleeve' of stomach 


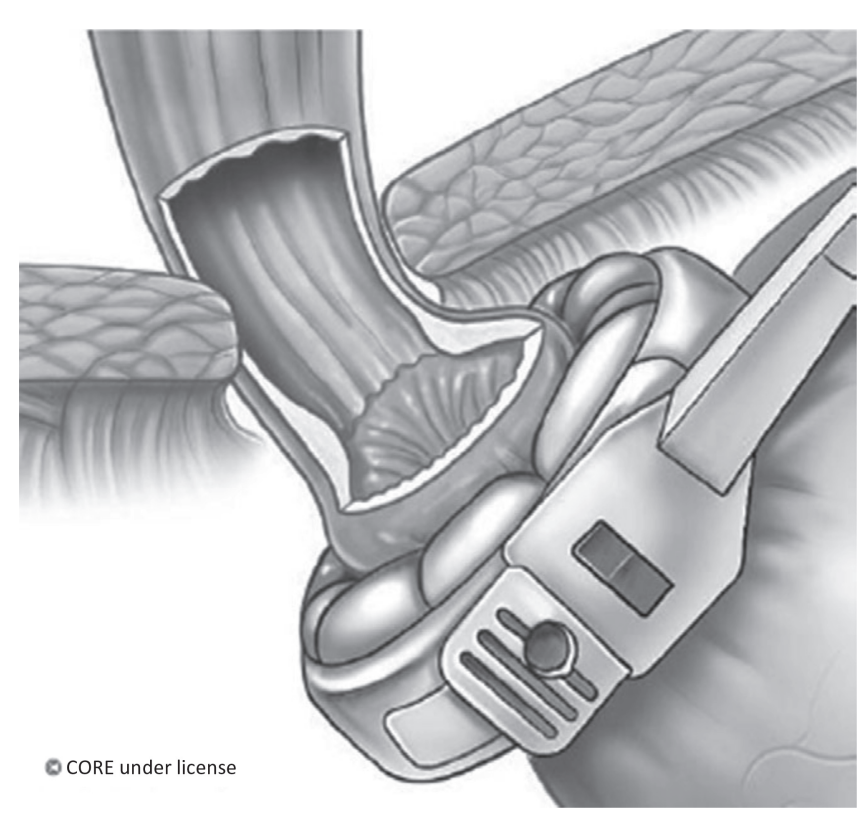

Fig 1. Laparoscopic adjustable gastric banding. An adjustable gastric band is placed at the very top of the stomach, with no significant gastric pouch above. Compression of vagal afferents within this area of gastric cardia is suggested to mediate the satiety effect. Reproduced with permission from John Wiley and Sons. ${ }^{2}$

along the lesser curve (Fig 3). 5,20,21 Originally the first step of the biliopancreatic diversion with duodenal switch (see below), sleeve gastrectomy has evolved into a staging procedure for super obese or high-risk patients..$^{2,5-7,20}$ It is also increasingly used as a standalone procedure. ${ }^{2,6,7}$

\section{Biliopancreatic diversion and biliopancreatic diversion with duodenal switch}

Biliopancreatic diversion (BPD) and biliopancreatic diversion with duodenal switch (BPD/DS) are malabsorptive operations that result in bypass of most of the small intestine. With BPD/ DS, a sleeve gastrectomy is performed, leaving the pylorus intact. The duodenum is then disconnected and the stomach anastomosed to the distal small bowel (the 'duodenal switch'), creating a short alimentary limb. The long biliopancreatic limb is then anastomosed to the ileum $75-100 \mathrm{~cm}$ proximal to the ileocaecal valve, so digestion and absorption occurs only in the short common channel. ${ }^{5,6,20}$ With BPD, a partial gastrectomy leaves a $400 \mathrm{ml}$ gastric pouch and the common channel is shortened to just $50 \mathrm{~cm}^{5}$

\section{Safety profile and complications}

Bariatric surgery is safe..$^{2,5,6,9,22-24}$ High-volume centres of excellence deliver bariatric surgery with inhospital mortality of $0.14 \%$ and 90 -day mortality of $0.35 \%$, which is comparable to that for cholecystectomy. ${ }^{6}$ Acute complications, including haemorrhage, obstruction, anastomotic leak, wound infection,

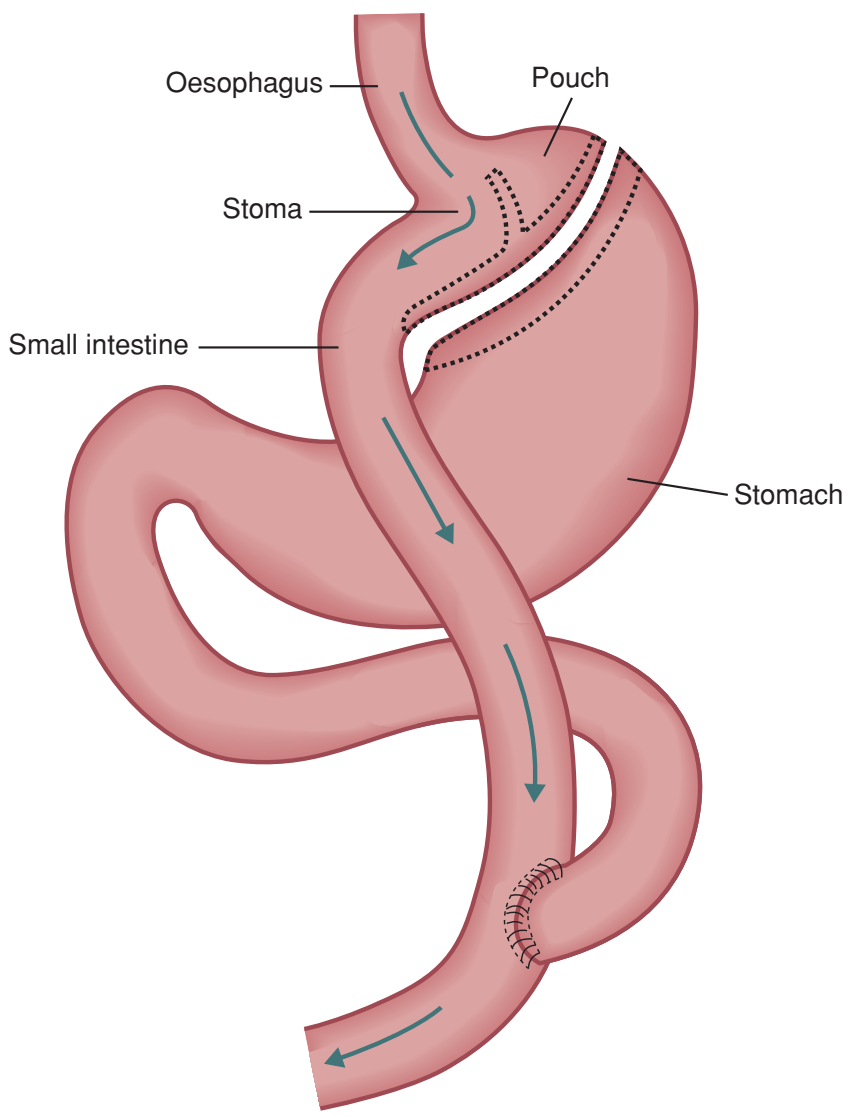

Fig 2. Roux-en-Y gastric bypass.

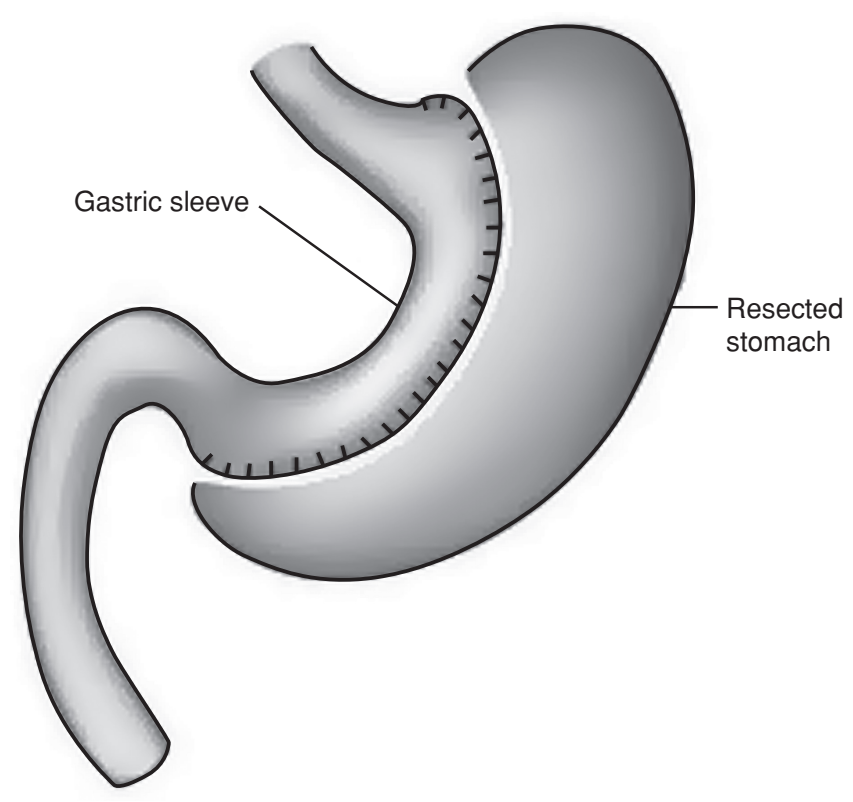

Fig 3. Sleeve gastrectomy. 
cardiac arrhythmias, pulmonary emboli, respiratory failure and rhabdomyolysis, occur in 5-10\% of patients. ${ }^{5,6,9,10,25}$

Long-term complications include internal hernias, anastomotic stenoses, marginal ulceration, fistulae, diarrhoea, dumping syndrome, gallstones, emotional disorders and nutritional deficiencies. ${ }^{5,6,13,15,20,25-27}$ Patients with LAGB can experience port problems, stomal obstruction, band slippage/erosion, pouch dilation, gastro-oesophageal reflux and oesophageal dilation. ${ }^{5,13,19,25}$ Malnutrition is a concern with BPD with or without DS. ${ }^{1,17}$ Long-term risks for sleeve gastrectomy are unknown. ${ }^{5}$

The Longitudinal Assessment of Bariatric Surgery ${ }^{22}$ reported overall 30 -day mortality of $0.3 \%$ for 4,610 patients having LAGB or RYGB for the first time, with $4.3 \%$ of patients having a major adverse outcome within 30 days. This was most frequent among patients having open RYGB (7.8\%). A meta-analysis involving 85,048 patients reported a total 30 -day mortality of $0.28 \%$ and a two-year mortality of a further $0.35 \% .{ }^{24}$ The most complex malabsorptive procedures had the highest perioperative mortality at $1.11 \%$. Mortality for gastric banding is between one in 2,000 and one in $5,700 .^{2}$

\section{Effects on weight, comorbidities and long-term mortality}

After RYGB, patients lose 60-70\% of their excess weight over two years, and this is largely durable. ${ }^{1,2,6,9,15,16,28}$ Weight loss is dependent on long-term compliance with dietary recommendations. ${ }^{2,5,6}$ Sugary, energy-dense foods and drinks can 'bypass the bypass'. After gastric banding, patients lose about 50\% (range $39 \%-59 \%$ ) of their excess weight at a slower rate, often continuing into the fifth year. ${ }^{1,2,5,6,9,19}$ Regular band adjustment is necessary. ${ }^{16}$ The Swedish Obese Subjects (SOS) study reported a reoperation or conversion rate of $31 \%$ for gastric banding and $17 \%$ for gastric bypass among patients followed for $\geqslant 10$ years, excluding operations for postoperative complications. ${ }^{16}$ However, at the centre in Melbourne, only about $10 \%$ of patients after LAGB need some revisional procedure, including band replacement, in the following 10 years. ${ }^{2}$ Biliopancreatic diversion with or without DS produces excess weight loss of $70.1 \%$; $^{9}$ sleeve gastrectomy produces initial excess weight loss of 55\%, but this may not be durable. ${ }^{2}$

Bariatric surgery also produces significant improvement in obesity-related comorbidities, with the most remarkable effect being resolution of type 2 diabetes (T2DM). A meta-analysis encompassing 22,094 patients reported complete remission of T2DM in $76.8 \%$ of patients, ${ }^{9}$ and a registry from the UK with data on 8,710 patients reported resolution of T2DM in $85.5 \%$ of patients. ${ }^{29}$ Major improvement often occurs within days after RYGB, before significant weight loss is achieved. ${ }^{5-7,17,18}$ After LAGB, improvement in T2DM occurs more slowly as a result of weight loss. ${ }^{2,7,19}$ Combined or malabsorptive procedures produce greater improvement than purely restrictive procedures. ${ }^{1,2,5,9,18}$ Diabetes less than three years in duration, no insulin requirement, milder obesity with $\mathrm{BMI}<40 \mathrm{~kg} / \mathrm{m}^{2}$ and weight loss $\geqslant 10 \%$ predict complete resolution of T2DM. ${ }^{18,19}$
Bariatric surgery is now advocated by some for the treatment of T2DM in patients with $\mathrm{BMI}<35 \mathrm{~kg} / \mathrm{m}^{2} .4,7,30,31$

Bariatic surgery effectively treats all other associated comorbidities: from steatohepatitis and pseudotumour cerebri to urinary incontinence. ${ }^{1-3,5,6,15}$ Meta-analysis showed that hyperlipidaemia improved in $\geqslant 70 \%$ of patients, hypertension resolved in $61.7 \%$ (and resolved or improved in $78.5 \%$ ) and obstructive sleep apnoea resolved in $83.6 \% .^{9}$ At five years, the risk of cardiovascular disease had decreased by $72 \% .^{3}$ The incidence of cancer also reduced markedly, ${ }^{6,15,16}$ as did the risk of developing new comorbid conditions. ${ }^{15}$ Long-term efficacy is well documented. $5,6,28$

At follow up after 10 years, the Swedish Obese Subjects (SOS) study showed a $29 \%$ reduction in adjusted all-cause mortality, primarily because of decreases in cancer and myocardial infarction. ${ }^{16}$ A retrospective cohort study of 7,925 patients after RYGB reported a $40 \%$ reduction in all-cause mortality during mean follow up of 7.1 years. ${ }^{23}$ Specific mortality decreased by $56 \%$ for coronary artery disease, by $92 \%$ for diabetes and by $60 \%$ for cancer. A large observational study, in which the vast majority of patients had undergone RYGB, reported an $89 \%$ risk reduction in five-year mortality. ${ }^{15}$

\section{Energy homeostasis and hormonal changes}

Weight loss after bariatric surgery is not explained by volume restriction and malabsorption alone. ${ }^{17}$ Indeed malabsorption is estimated to account for only $5 \%$ of the weight loss following standard RYGB. ${ }^{17}$ Bariatric surgery causes significant changes in the neurohormonal profile, which contributes to sustained weight loss through changes in appetite, satiety, food preferences and eating patterns and explains the remarkable effect on T2DM. ${ }^{2,5-7,17,18}$

\section{The hypothalamus ${ }^{32}$}

Hormonal signals provide information about energy status to the hypothalamus. Adipokines are secreted by adipose tissue and enterokines by the gut. Incretins are enterokines that stimulate release of insulin after food intake. ${ }^{18}$ Two hypothalamic circuits influence food intake, and both contribute to acquisition and storage of nutrient energy. The homeostatic circuit increases appetite and locomotion in response to energy shortage. The hedonic circuit is engaged at stable weight plateaus in association with increases in body fat. It heightens finickiness to taste of food. Obese animals overeat palatable food but undereat bland foods and lose weight. In our current obesogenic environment, the hedonic circuit facilitates the seeking of energy-dense foods uncoupled from energy status.

\section{Enterokines}

Ghrelin, ${ }^{33}$ which is mostly synthesised in the stomach, is a potent appetite stimulator involved in hunger and meal initiation. Circulating levels are inversely proportional to BMI and respond 
to changes in body weight. Ghrelin enhances gut motility and speeds gastric emptying. ${ }^{17}$ It promotes lipid accumulation and weight gain, favouring glucose utilisation. It also inhibits insulin secretion and impairs glucose tolerance. ${ }^{18}$ Levels of ghrelin reported after bariatric surgery have been variable, which may be due to differences in surgical techniques and research methods.,18 Overall, the trend is for a decrease in ghrelin levels after RYGB and an increase after gastric banding. ${ }^{717}$ Sleeve gastrectomy, which removes most of the ghrelin-producing stomach, reduces levels of ghrelin.

Peptide YY (PYY) ${ }^{34}$ is secreted postprandially by L cells in the pancreas, small intestine and colon. It suppresses appetite and promotes satiety via signalling actions in the brain. It also delays gastric emptying (the ileal brake) and enhances insulin sensitivity. ${ }^{7}$ Secretion of PYY generally corresponds to the energy ingested, although it may vary depending on the macronutrient content. ${ }^{17}$ Interestingly, levels also correlate positively with exercise intensity, with resulting decreases in food intake.

Glucagon-like peptide 1 (GLP-1) is co-secreted postprandially with PYY in the distal intestine. ${ }^{17} \mathrm{~A}$ powerful incretin, GLP-1 potentiates glucose-stimulated insulin secretion, enhances $\beta$-cell growth and survival, inhibits glucagon release and enhances all steps of insulin biosynthesis. ${ }^{7,17}$ It also slows gastric emptying to produce greater gastric distension and helps regulate appetite and body weight. ${ }^{7,17}$ Obese individuals have lower levels of PYY and GLP-1, and levels are decreased further in patients with diabetes. ${ }^{5,17,18}$

Two hypotheses exist to explain the hormonal and metabolic effects of the RYGB:

- the hindgut hypothesis

- the foregut exclusion theory.

The hindgut hypothesis postulates that after RYGB and malabsorptive procedures, rapid nutrient delivery to the distal gut $\mathrm{L}$ cells and their increased exposure to incompletely digested nutrients lead to an early and exaggerated PYY and GLP-1 response, contributing to early satiety, reduced meal size and early resolution of T2DM.,17,18 Ileal transposition studies provide strong evidence for this. Interposition of an ileal segment into the proximal gut in rodents produced exaggerated PYY, GLP-1 and enteroglucagon responses, reduced food intake, weight loss, improved insulin sensitivity and overall improved glucose homeostasis. ${ }^{7}$

The foregut exclusion theory proposes that exclusion of the duodenum and proximal jejunum after RYGB is the mechanism that mediates the effects of bariatric surgery. ${ }^{7,18}$ However, duodenal-jejunal bypass experiments in rats supporting this theory are compounded by the accompanying pyloric disruption that results in accelerated gastric emptying and rapid nutrient delivery to the hindgut. ${ }^{7}$ The endoluminal duodenaljejunal sleeve also accelerates gastric emptying by abolishing duodenal osmoreceptor control of pyloric contraction. ${ }^{7}$ This $60 \mathrm{~cm}$-long sleeve prevents nutrient contact with the duodenum and proximal jejunum, while biliary and pancreatic secretions flow outside the sleeve, delaying digestion. ${ }^{35}$
A possible mediator of the foregut exclusion theory is the gastric inhibitory polypeptide or glucose-dependent insulinotropic polypeptide (GIP), which is secreted by K cells in the duodenum in response to nutrient absorption. ${ }^{18,39,40}$ In addition to its incretin action, GIP promotes lipogenesis ${ }^{41}$ with GIP receptor knockout mice protected against diet-induced obesity and insulin resistance, ${ }^{39}$ while antagonism of the GIP receptor improves glucose tolerance and insulin sensitivity and partially corrects pancreatic islet hypertrophy and $\beta$-cell hyperplasia. ${ }^{40}$ Levels of GIP are suppressed after malabsorptive procedures. ${ }^{18,41}$

\section{Adipokines}

Adiponectin ${ }^{17,36,37}$ is synthesised primarily in adipose tissue, with levels inversely correlated with BMI. It is an important insulin sensitiser, and hypoadiponectinaemia causes insulin resistance and T2DM. Adiponectin also possesses antiatherogenic, anti-inflammatory and cardioprotective properties and may act centrally to modulate food intake and energy expenditure. Weight loss following bariatic surgery increases levels of adiponectin.

Leptin $^{38,32}$ is secreted by adipose tissue and regulates body weight via its action on the hypothalamus. It increases nocturnally to stimulate lipolysis but also increases postprandially to induce anorexia. In addition, leptin plays an important role in glucose homeostasis. Levels of leptin are proportional to body fat, with starvation or energy shortage activating the homeostatic mechanism in the hypothalamus to restore energy balance. However, leptin resistance develops in obesity. Weight loss from bariatic surgery reduces leptin levels. ${ }^{17}$

Many other enterokines and adipokines exist, some of which may also play a part in producing and sustaining weight loss or diabetes remission after bariatric surgery. ${ }^{17,18}$ Understanding the mechanisms of action of bariatric surgery will help realise therapeutic benefits by pharmacological means. ${ }^{6,7,19}$

\section{Nutritional deficiencies}

Nutritional deficiency is common after bariatric surgery and the risk increases from LAGB through SG and RYGB to BPD with or without DS..$^{20,26,31}$ The problem is heightened by the fact that micronutrient deficiencies are already highly prevalent in obese patients before surgery. ${ }^{21}$ After surgery, patients are at particular risk of deficiencies in vitamins $B_{1}, B_{12}, C, A$ and $D$ and folic acid, as well as iron, calcium and protein. ${ }^{20,26}$ Lifelong prophylactic supplementation is often necessary, and regular monitoring is essential. ${ }^{26}$ Investigation of clinical syndromes resulting from malnutrition can be challenging.

\section{Anaemia $^{20,27}$}

After bariatric surgery, patients are prone to iron deficiency because of intestinal bypass, pouch hypoacidity and intolerance of red meat. Obesity creates a state of chronic inflammation that 
can contribute to anaemia. Anaemia can also be caused by deficiencies in folate, vitamin $B_{12}$, vitamin $\mathrm{E}$ (haemolytic anaemia), copper (anaemia and neutropenia), vitamin $A$ and zinc. In refractory anaemia, gastrointestinal blood loss must be considered. Bleeding in the excluded stomach, duodenum or biliopancreatic limb is problematic as the usual endoscopic access route is no longer available.

\section{Neurological problems $\boldsymbol{s}^{6,20}$}

Neurological symptoms can result from deficiencies in thiamine, vitamin $\mathrm{B}_{12}$, niacin, vitamin $\mathrm{E}$ and copper or from hypocalcaemia secondary to vitamin $\mathrm{D}$ deficiency. Clinical syndromes includes Wernicke's encephalopathy, peripheral neuropathy, dry beriberi, neuropsychiatric beriberi, pellagra, ataxia, spasticity, myelopathy, muscle weakness, posterior column signs and ptosis.

\section{Oedema $^{6,20}$}

Patients with oedema may have underlying heart failure, which can also be due to wet beriberi (thiamine deficiency) or selenium deficiency. Hypoalbuminaemia may be caused by liver cirrhosis secondary to steatohepatitis; severe protein/calorie malnutrition; kwashiorkor; and diarrhoea secondary to bacterial overgrowth, malabsorption of bile salts and niacin deficiency.

\section{Eye, skin and hair problems ${ }^{20}$}

Vitamin A deficiency causes difficulties with nocturnal vision and reduced visual acuity. Vitamin E deficiency can cause retinopathy. Thiamine deficiency can present with blurred or double vision. Dry skin, pruritus and rash can be caused by deficiencies in vitamin A, niacin, riboflavin, zinc and essential fatty acids. Hair changes can be due to zinc deficiency or protein malnutrition.

\section{Pregnancy after bariatric surgery}

About half of patients undergoing bariatric surgery are women of childbearing age, ${ }^{8}$ which introduces specific concerns. Obesity is strongly associated with infertility ${ }^{8}$ and increases the risk of obstetric complications. ${ }^{8}$ Yet the effects of rapid weight loss and potential malnutrition in pregnant patients are of concern.

Bariatric surgery improves fertility ${ }^{42,43}$ and reduces the incidence of obesity-related complications such as gestational hypertension, gestational diabetes, pre-eclampsia and foetal macrosomia when compared with obese controls. The effect on premature delivery, miscarriage, intrauterine growth retardation, low birth weight and neural tube defects and the need for caesarean section are unclear. ${ }^{8,43}$ Maternal surgical weight loss reduces the prevalence of obesity and cardiometabolic risk factors in offspring until the adolescent years. ${ }^{42}$

Pregnancy seems to have little effect on the surgically induced weight loss. ${ }^{8}$ Patients are generally advised to delay pregnancy until after the period of maximal weight loss (12-18 months). ${ }^{8}$ Extra vigilance in preconception, antenatal and obstetric care is required.

\section{Conclusion}

In summary, bariatric surgery is a safe and effective treatment for severe obesity and its associated comorbidities. It is particularly effective in the treatment of T2DM. Neurohormonal changes that affect appetite, satiety, glucose homeostasis and long-term energy balance contribute to its long-term efficacy. Two hypotheses exist to explain how hormonal changes produce these effects, and both may contribute. Patient adherence to postsurgical aspects of management is very important. Pregnancy after bariatric surgery brings additional considerations. Finally, the indications for bariatric surgery are being widened.

\section{Acknowledgements}

Dr Keng Ngee Hng is a specialty registrar in gastroenterology and has previously submitted part of this work for her Master of Science in gastroenterology (Salford University). Dr Yeng S Ang is the educational supervisor for Dr Hng and has refined the ideas, concepts and layout of the previous work. Recent updates are also included within this paper.

\section{References}

1 Chang J, Wittert G. Effects of bariatric surgery on morbidity and mortality in severe obesity. Int J Evid Based Healthc 2009;7:43-48.

2 O'Brien PE. Bariatric surgery: mechanisms, indications and outcomes. J Gastroenterol Hepatol 2010;25:1358-65.

3 Ashrafian H, Carel W le Roux, Ara D, Thanos A. Effects of bariatric surgery on cardiovascular function. Circulation 2008;118:2091-102.

4 Lakdawala M, Bhasker A. Report: Asian Consensus Meeting on Metabolic Surgery. Recommendations for the use of bariatric and gastrointestinal metabolic surgery for treatment of obesity and type ii diabetes mellitus in the Asian population. Obes Surg 2010;20:929-36.

5 Smith BR, Schauer P, Nguyen NT. Surgical approaches to the treatment of obesity: bariatric surgery. Endocrinol Metab Clin North Am 2008;37:943-64.

6 Pories WJ. Bariatric surgery: risks and rewards. J Clin Endocrinol Metab 2008;93(11 Suppl 1):S89-96.

7 Karra E, Yousseif A, Batterham RL. Mechanisms facilitating weight loss and resolution of type 2 diabetes following bariatric surgery. Trends Endocrinol Metab 2010;21:337-44.

8 Beard JH, Bell RL, Duffy AJ. Reproductive considerations and pregnancy after bariatric surgery: current evidence and recommendations. Obes Surg 2008;18:1023-7.

9 Buchwald H, Avidor Y, Braunwald E et al. Bariatric surgery: a systematic review and meta-analysis. JAMA 2004;292:1724-37.

10 Kokkinos P, Moutsatsos G. Obesity and cardiovascular disease: the role of diet and physical activity. J Cardiopulm Rehabil 2004;24:197-204.

11 Jordan J, Schlaich M, Redon J et al. European Society of Hypertension Working Group on Obesity: obesity drugs and cardiovascular outcomes. J Hypertens 2011;29:189-93.

12 Elangbam CS. Review paper: current strategies in the development of anti-obesity drugs and their safety concerns. Vet Pathol 2009;46:10-24.

13 Cannon-Diehl MR. Emerging issues for the postbariatric surgical patient. Critic Care Nurs Q 2010;33:361-70. 
14 National Institute for Health and Clinical Excellence. Obesity: guidance on the prevention, identification, assessment and management of overweight and obesity in adults and children. Clinical guideline 43. London: NICE, 2006.

15 Christou NV, Sampalis JS, Liberman M et al. Surgery decreases longterm mortality, morbidity, and health care use in morbidly obese patients. Ann Surg 2004;240:416-24.

16 Sjostrom L, Narbro K, Sjostrom D et al. Effects of bariatric surgery on mortality in Swedish obese subjects. N Engl J Med 2007;357:741-52.

17 Ochner CN, Gibson C, Shanik M, Goel V, Geliebter A. Changes in neurohormonal gut peptides following bariatric surgery. Int J Obes (London) 2011;35:153-66.

18 Vetter ML, Cardillo S, Rickels MR, Iqbal N. Narrative review: effect of bariatric surgery on type 2 diabetes mellitus. Ann Intern Med 2009;150:94-103.

19 Pinkney J. Bariatric surgery for diabetes: gastric banding is simple and safe. Br J Diabetes Vasc Dis 2010;10:139-42.

20 Koch TM, Finelli FC. Postoperative metabolic and nutritional complications of bariatric surgery. Gastroenterol Clin North Am 2010;39:10924.

21 British Obesity Surgery Patient Association, 2012. www.bospa.org [Accessed 9 March 2012].

22 Longitudinal Assessment of Bariatric Surgery (LABS) Consortium. Perioperative safety in the longitudinal assessment of bariatric surgery. N Engl J Med 2009;361:445-54.

23 Adams TD, Gress RE, Smith SC et al. Long-term mortality after gastric bypass surgery. N Engl J Med 2007;357:753-61.

24 Buchwald H, Estok R, Fahrbach K, Banel D, Sledge I. Trends in mortality in bariatric surgery: a systematic review and meta-analysis. Surgery 2007;142:621-32.

25 Monkhouse SJW, Morgan JDT, Norton SA. Complications of bariatric surgery: presentation and emergency management - a review. Ann $R$ Coll Surg Engl 2009;91:280-6.

26 Schweitzer DH, Posthuma EF. Prevention of vitamin and mineral deficiencies after bariatric surgery: evidence and algorithms. Obes Surg 2008;18:1485-8.

27 von Drygalski A, Andris DA. Anemia after bariatric surgery: more than just iron deficiency. Nutr Clin Pract 2009;24:217-26.

28 Sjostrom L, Lindroos AK, Peltonen M et al. Lifestyle, diabetes, and cardiovascular risk factors 10 years after bariatric surgery. $N$ Engl J Med 2004;351:2683-93.

29 Welbourn P, Fiennes A, Kinsman R, Walton P. United Kingdom National Bariatric Surgery Registry: first registry report to March 2010. Henley-on-Thames: Dendrite Clinical Systems, 2010:1-215.

30 Rubino F, Kaplan LM, Schauer PR, Cummings DE. The Diabetes Surgery Summit consensus conference: recommendations for the evaluation and use of gastrointestinal surgery to treat type 2 diabetes mellitus. Ann Surg 2010;251:399-405.

31 International Diabetes Federation. Bariatric surgical and procedural interventions in the treatment of obese patients with type 2 diabetes: a position statement from the International Diabetes Federation Taskforce on Epidemiology and Prevention. Brussels: IDF, 2011. Available at: www. idf.org (accessed 30 July 2012).

32 Borer KT. Nonhomeostatic control of human appetite and physical activity in regulation of energy balance. Exerc Sport Sci Rev 2010;38:114-21.

33 Scerif M, Goldstone AP, Korbonits M. Ghrelin in obesity and endocrine diseases. Mol Cell Endocrinol 2011;340:15-25.

34 Nguyen AD, Herzog H, Sainsbury A. Neuropeptide Y and peptide YY: important regulators of energy metabolism. Curr Opin Endocrinol Diabetes Obes 2011;18:56-60.

35 Gersin KS, Keller JE, Stefanidis D et al. Duodenal-jejunal bypass sleeve: a totally endoscopic device for the treatment of morbid obesity. Surg Innov 2007;14:275-8.

36 Szmitko PE, Teoh H, Stewart DJ, Verma S. Adiponectin and cardiovascular disease: state of the art? Am J Physiol Heart Circ Physiol 2007;292:H1655-63.

37 Ziemke F, Mantzoros CS. Adiponectin in insulin resistance: lessons from translational research. Am J Clin Nutrition 2010;9:258S-61S.

38 Morton GJ. Hypothalamic leptin regulation of energy homeostasis and glucose metabolism. J Physiol 2007;583:437-43.

39 Miyawaki K, Yamada Y, Ban N et al. Inhibition of gastric inhibitory polypeptide signaling prevents obesity. Nat Med 2002;8:738-42.

40 Victor AG, Irwin N, Green BD et al. Chemical ablation of gastric inhibitory polypeptide receptor action by daily (Pro3) GIP administration improves glucose tolerance and ameliorates insulin resistance and abnormalities of islet structure in obesity-related diabetes. Diabetes 2005;54:2436-46.

41 Flatt PR, Day C, Bailey CJ. Bariatric surgery: to treat diabesity. $\mathrm{Br} \mathrm{J}$ Diabetes Vasc Dis 2009;9:103-7.

42 Merhi Z, Jindal S, Pollack SE, Lieman HJ. Pregnancy following bariatric surgery. Expert Rev Obstet Gynecol 2011;6:57-67.

43 Guelinckx I, Devlieger R, Vansant G. Reproductive outcome after bariatric surgery: a critical review. Hum Reprod Update 2009;15: 189-201.

Address for correspondence: Dr KN Hng, 4 Fern Close, Shevington, Wigan WN6 8BL.

Email: keng_ngee@hotmail.com 